九州大学学術情報リポジトリ

Kyushu University Institutional Repository

\title{
Denoising of multi-modal images with PCA self- cross bilateral filter
}

Qiu, Yu

College of Communication Engineering, Chongqing University

Urahama, Ki ichi

Faculty of Design, Kyushu University

http://hdl. handle. net/2324/26351

出版情報: IEICE Transactions on Fundamentals of Electronics, Communications and Computer Sciences. E93.A (9)，pp.1709-1712，2010-09. 電子情報通信学会

バージョン:

権利関係: (C) 2010 The Institute of Electronics, Information and Communication Engineers 


\title{
LETTER \\ Denoising of Multi-Modal Images with PCA Self-Cross Bilateral Filter
}

\author{
Yu QIU ${ }^{\dagger}$, Nonmember and Kiichi URAHAMA ${ }^{\dagger \dagger \mathrm{a})}$, Member
}

\begin{abstract}
SUMMARY We present the PCA self-cross bilateral filter for denoising multi-modal images. We firstly apply the principal component analysis for input multi-modal images. We next smooth the first principal component with a preliminary filter and use it as a supplementary image for cross bilateral filtering of input images. Among some preliminary filters, the undecimated wavelet transform is useful for effective denoising of various multi-modal images such as color, multi-lighting and medical images.

key words: multi-modal image, bilateral filter, cross bilateral filter, principal component analysis
\end{abstract}

\section{Introduction}

The self-cross bilateral filter (SCBF) [1] has recently been proposed for boosting noise reduction capability of the bilateral filter (BF) [2]. The SCBF is a special type of the cross bilateral filter (CBF) [3]. In the CBF, the filter coefficients are computed from a supplementary image. In the SCBF, the supplementary image is made with a supplementary filter preliminarily applied to an inputted target image.

In this letter, we extend the SCBF to multi-modal images. In the BF for multi-modal images, its filter coefficients are usually computed with integration of all component images and commonly used for filtering them. In this integration, each component image is conventionally weighted equally. However noise intensity differs generally for each component image, hence adaptive weights are preferable for effective denoising of multi-modal images. For instance, in color images, the blue channel generally contains noises larger than the red and green channels. Practical noise intensity is, however difficult to estimate because it depends on cameras and photographing conditions. The estimation is further difficult for general multi-modal images such as medical or remote sensing images.

From these observations, we propose, in this letter, a SCBF utilizing a supplementary image computed with the principal component analysis (PCA) for denoising multimodal images. The PCA is a weighted average of all components. As is well-known, averaging generally reduces noises. However multi-modal images cannot be simply averaged because polarity differs for each component. The PCA automatically integrates all components with maximiz-

Manuscript received March 11, 2010.

Manuscript revised May 17, 2010.

$\dagger$ The author is with the College of Communication Engineering, Chongqing University, Chongqing, 400044 China.

${ }^{\dagger \dagger}$ The author is with the Faculty of Design, Kyushu University, Fukuoka-shi, 815-8540 Japan.

a)E-mail: urahama@ design.kyushu-u.ac.jp

DOI: 10.1587/transfun.E93.A.1709 ing the variance of outputs, which increases the variance of signal components and hence relatively reduces noise intensity in an output image. In the proposed method, we firstly compute the first principal component in the PCA. We next smooth it using a preliminary filter. We then denoise every component in a multi-modal image with the CBF utilizing the smoothed principal component as a supplementary image.

\section{PCA Self-Cross Bilateral Filter}

Let a multi-modal image be composed of $n$ component images. We denote the $k$-th component value of pixel $(i, j)$ as $d_{i j k}$, For instance, in color images, $n=3$ and components are red, green and blue channels: $d_{i j 1}=R_{i j}, d_{i j 2}=$ $G_{i j}, d_{i j 3}=B_{i j}$.

The output of BF is generally expressed as

$$
f_{i j k}=\frac{\sum_{l=-p}^{p} \sum_{m=-p}^{p} s_{l m} w_{i j k l m} d_{i+l, j+m, k}}{\sum_{l=-p}^{p} \sum_{m=-p}^{p} s_{l m} w_{i j k l m}}
$$

where $s_{l m}=e^{-\left(l^{2}+m^{2}\right) / \sigma_{d}^{2}}$. There can be considered several variations for $w_{i j k l m}$ as follows (see also Fig. 1):

1) IBF (Fig. 1(a))

If we apply BF to every component $k$ independently, then $w_{i j k l m}=e^{-\left(d_{i j k}-d_{i+l, j+m, k}\right)^{2} / \sigma_{r}^{2}}$ is different for each $k$. We call this method the independent BF (IBF).

2) EBF (Fig. 1(b))

Another simple version is using $w_{i j k l m}=w_{i j l m}=$ $e^{-\sum_{k}\left(d_{i j k}-d_{i+l, j+m, k}\right)^{2} / \sigma_{r}^{2}}$ for every component $k$ commonly. We call this the Euclidean BF (EBF).

3) PCA-CBF (Fig. 1(c))

In the above IBF and EBF, $w_{i j k l m}$ is computed directly from an input image. We refine this as we firstly apply PCA to an input image and compute the first principal component $g_{i j}$ and use it as a supplementary image for CBF.

The PCA starts with the computation of average of each component as $\bar{d}_{k}=\sum_{i} \sum_{j} d_{i j k} / T$ where $T$ is the total number of pixels. We next compute the covariance matrix $C=\left[c_{k k^{\prime}}\right]\left(k, k^{\prime} \in[1, n]\right)$ where $c_{k k^{\prime}}=\sum_{i} \sum_{j}\left(d_{i j k}-\right.$ $\left.\bar{d}_{k}\right)\left(d_{i j k^{\prime}}-\bar{d}_{k^{\prime}}\right) / T$. The first principal component is its first eigenvector $\left[u_{1}, \ldots, u_{n}\right]^{T}$. The vector of multi-modal image $\left[d_{i j 1}-\bar{d}_{1}, \ldots, d_{i j n}-\bar{d}_{n}\right]^{T}$ is then projected to this eigenvector as $g_{i j}=\sum_{k} u_{k}\left(d_{i j k}-\bar{d}_{k}\right)$ which is used as a supplementary 


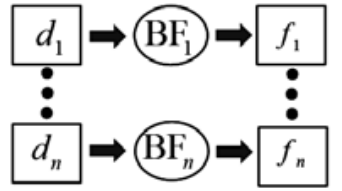

(a) IBF

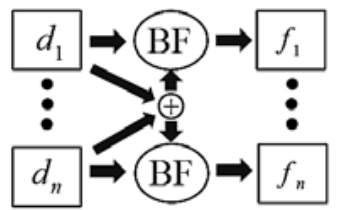

(b) $\mathrm{EBF}$

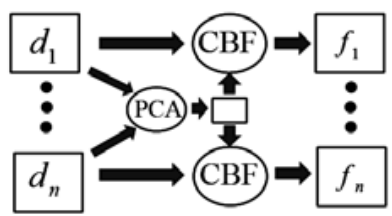

(c) PCA-CBF

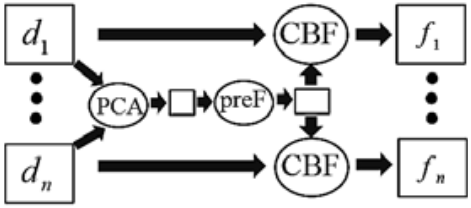

(d) PCA-preF-CBF

Fig. 1 Diagrams of filters.

image. We call this method the PCA-CBF.

4) PCA-preF-CBF (Fig. 1(d))

This PCA-CBF can be further improved by smoothing $g_{i j}$ before used as a supplementary image. We can use any filter, BF or wavelet filter, for this preliminary smoothing of $g_{i j}$. We denote the output of this preliminary filter, i.e. smoothed $g_{i j}$, as $h_{i j}$ and use $w_{i j k l m}=w_{i j l m}=e^{-\left(h_{i j}-h_{i+l, j+m}\right)^{2} / \sigma_{r}^{2}}$ for every component image commonly. We call this the PCA-preF-CBF where the preliminary filter preF is practically BF, wavelet transform filter and so on.

\section{Experiments}

We have experimented the above filters for a color image which is a typical multi-modal image, a set of photographs of a leaf lighted from three different directions and two MR images of brains. We have evaluated the PSNR of filter outputs for various intensity of added Gaussian noises. We added mutually independent noises of the same standard deviation to each component image.

We have conducted many experiments by varying all parameters little by little and we set the parameters in each filter to its best values which vary with the magnitude of noises.

\subsection{Color Image}

We firstly experimented with a color image "lena" of which
Table 1 PSNR (dB) for color "lena."

\begin{tabular}{|r|r|r|r|r|r|}
\hline$\sigma$ & IBF & EBF & P-CBF & P-BF-CBF & P-WT-CBF \\
\hline 3 & 40.81 & 41.91 & $\mathbf{4 2 . 1 5}$ & 42.02 & 41.61 \\
\hline 5 & 37.81 & 38.96 & 39.26 & $\mathbf{3 9 . 3 3}$ & 38.84 \\
\hline 7 & 35.99 & 37.09 & 37.43 & $\mathbf{3 7 . 5 2}$ & 37.23 \\
\hline 10 & 34.12 & 35.18 & 35.58 & $\mathbf{3 5 . 8 5}$ & 35.73 \\
\hline 15 & 32.05 & 32.98 & 33.46 & $\mathbf{3 4 . 1 8}$ & 34.07 \\
\hline 20 & 30.62 & 31.44 & 31.94 & $\mathbf{3 2 . 9 2}$ & 32.92 \\
\hline 25 & 29.52 & 30.20 & 30.73 & $\mathbf{3 1 . 9 9}$ & 31.94 \\
\hline 30 & 28.59 & 29.20 & 29.72 & $\mathbf{3 1 . 1 1}$ & 31.11 \\
\hline 35 & 27.87 & 28.36 & 28.86 & $\mathbf{3 0 . 4 0}$ & 30.37 \\
\hline 40 & 27.16 & 27.56 & 28.01 & 29.60 & $\mathbf{2 9 . 6 2}$ \\
\hline
\end{tabular}

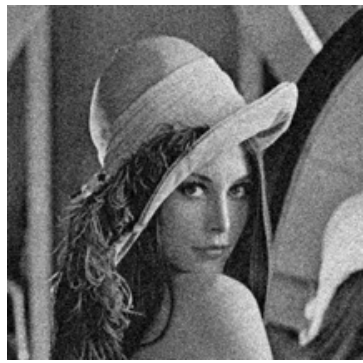

(a) input

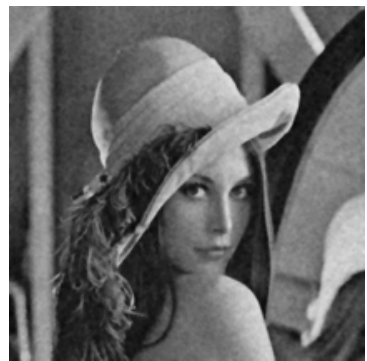

(c) $\mathrm{EBF}$

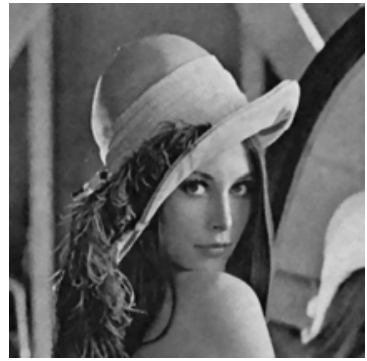

(e) PCA-BF-CBF

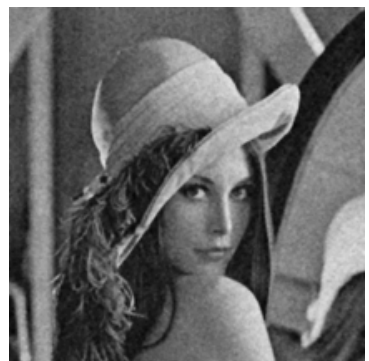

(b) IBF

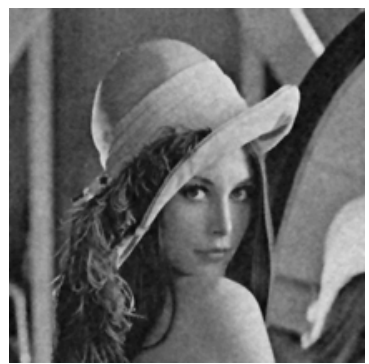

(d) PCA-CBF

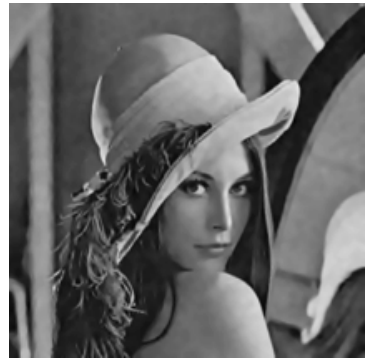

(f) PCA-UWT-CBF
Fig. 2 Input and outputs of filters.

PSNR of some filters are shown in Table 1 where $\sigma$ is the standard deviation of noises independently added to each component R, G, and B. In Table 1, "P-CBF" is abbreviation of PCA-CBF, "P-BF-CBF" is also PCA-BF-CBF and "PWT-CBF" is PCA-UWT-CBF where UWT denotes the undecimated wavelet transform [4]. The largest PSNR among these filters is highlighted with bold font. The PCA-BFCBF is broadly top-ranked while the PCA-UWT-CBF is next with close scores.

All images are in color however this print is monochromatic. In such monochromatic printing, gray-scale im- 


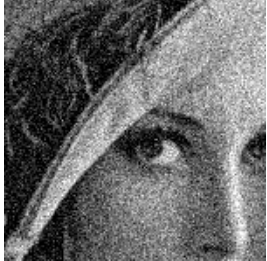

(a) input

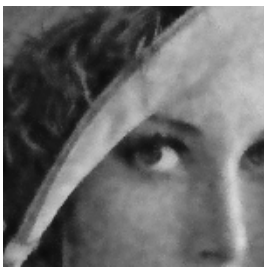

(c) $\mathrm{EBF}$

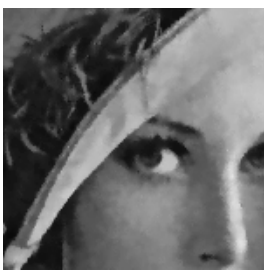

(e) PCA-BF-CBF

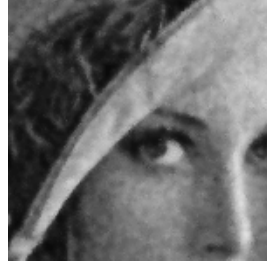

(b) IRF

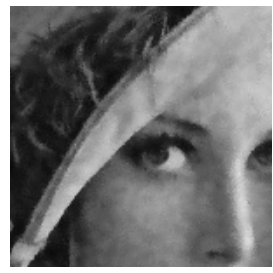

(d) $\mathrm{PCA}-\mathrm{CBF}$

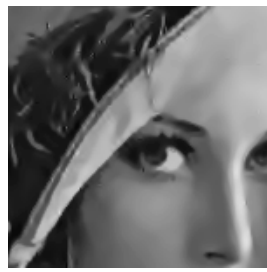

(f) PCA-UWT-CBF
Fig. 3 Enlarged parts in Fig. 2.

ages are popularly displayed, however in our experiment noises in each channel are mutually independent, hence the weighted averaging for transforming color image to its grayscale reduces noise intensity. Therefore instead of popular grayscale images, we show green-component images in Fig. 2 where (a) is an input image with $\sigma=30$, (b) is the output of IBF, (c) is EBF, (d) is PCA-CBF, (e) is PCA-BF-CBF and (f) is PCA-UWT-CBF. Because these images are downsized from $512 \times 512$ to these small printed sizes, noises are smoothed by this down-sizing and difference between these images is difficult to evaluate visually. Therefore we append enlarged display of parts in these images for the ease of comparison of their quality (remained noise level) in Fig. 3.

We have also experimented the CBF using the luminance as a supplementary image, however its performance is worse than the PCA-CBF.

\subsection{Multi-Modal Image}

We have next experimented with the photographs of a leaf lighted from three directions shown in Fig. 4(a) and two MR images of brain in Fig. 4(b). The PSNR for Fig. 4(a) is shown in Table 2 and that for Fig. 4(b) is shown in Table 3.

For these multi-modal images, the EBF is best when $\sigma$ is small while the PCA-UWT-CBF becomes top-ranked when noises increase. This may be due to that the singlepass of basic BF is sufficient when noise intensity is low and the multi-pass of BF such as the CBF degrades PSNR. This is due to that parameters are adjusted to their optimal values
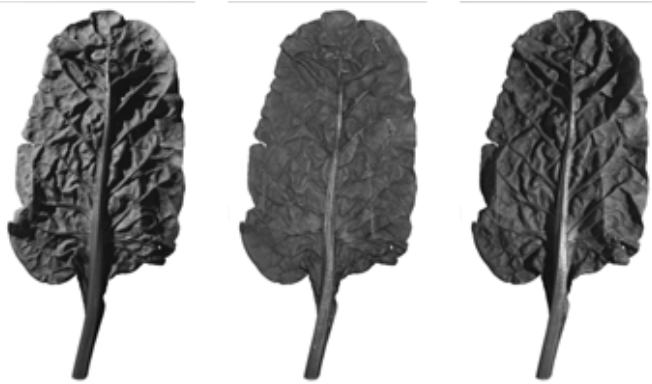

(a) leaf
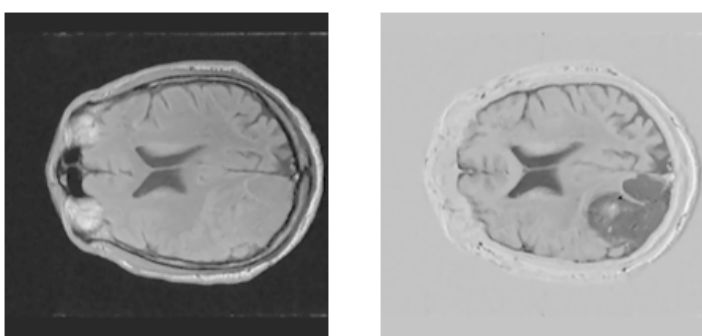

(b) brain

Fig. 4 Multi-modal images.

Table 2 PSNR $(\mathrm{dB})$ for "leaf."

\begin{tabular}{|r|r|r|r|r|r|}
\hline$\sigma$ & IBF & EBF & P-CBF & P-BF-CBF & P-WT-CBF \\
\hline 3 & 42.41 & $\mathbf{4 2 . 5 5}$ & 40.24 & 40.70 & 41.36 \\
\hline 5 & 39.27 & $\mathbf{3 9 . 4 5}$ & 37.75 & 38.03 & 38.49 \\
\hline 7 & 37.30 & $\mathbf{3 7 . 4 9}$ & 36.42 & 36.61 & 36.96 \\
\hline 10 & 35.26 & $\mathbf{3 5 . 4 3}$ & 34.91 & 34.96 & 35.34 \\
\hline 15 & 32.52 & 33.22 & 32.96 & 33.12 & $\mathbf{3 3 . 3 1}$ \\
\hline 20 & 30.30 & 31.44 & 30.65 & 31.61 & $\mathbf{3 1 . 7 4}$ \\
\hline 25 & 28.55 & 30.02 & 29.26 & 30.63 & $\mathbf{3 0 . 8 8}$ \\
\hline 30 & 27.62 & 28.89 & 28.20 & 30.07 & $\mathbf{3 0 . 1 7}$ \\
\hline 35 & 26.82 & 27.93 & 27.35 & 29.33 & $\mathbf{2 9 . 5 2}$ \\
\hline 40 & 26.13 & 27.08 & 26.63 & 28.81 & $\mathbf{2 9 . 0 4}$ \\
\hline
\end{tabular}

Table 3 PSNR (dB) for "brain."

\begin{tabular}{|r|r|r|r|r|r|}
\hline$\sigma$ & IBF & EBF & P-CBF & P-BF-CBF & P-WT-CBF \\
\hline 3 & 43.45 & $\mathbf{4 3 . 7 2}$ & 42.31 & 42.51 & 43.29 \\
\hline 5 & 40.33 & $\mathbf{4 0 . 6 0}$ & 39.68 & 39.74 & 40.50 \\
\hline 7 & 38.36 & 38.58 & 37.83 & 38.03 & $\mathbf{3 8 . 6 9}$ \\
\hline 10 & 36.24 & 36.37 & 35.88 & 36.33 & $\mathbf{3 6 . 8 8}$ \\
\hline 15 & 33.90 & 33.78 & 33.75 & 34.50 & $\mathbf{3 4 . 8 7}$ \\
\hline 20 & 32.41 & 32.32 & 32.41 & 33.13 & $\mathbf{3 3 . 5 9}$ \\
\hline 25 & 30.98 & 31.31 & 31.36 & 32.15 & $\mathbf{3 2 . 5 5}$ \\
\hline 30 & 30.49 & 30.64 & 30.61 & 31.48 & $\mathbf{3 1 . 8 1}$ \\
\hline 35 & 29.36 & 29.78 & 29.83 & 30.67 & $\mathbf{3 1 . 0 1}$ \\
\hline 40 & 29.00 & 29.43 & 29.41 & 30.27 & $\mathbf{3 0 . 6 2}$ \\
\hline
\end{tabular}

in our experiments. It is commonly observed in repetitive $\mathrm{BF}$ that PSNR decreases with iteration if the value of parameters is optimal and noise-level is low. If noise becomes large, then iteration of BF such as CBF becomes effective. Superiority of EBF over IBF may be due to the averaging of three components in EBF is effective for reducing noises.

An enlarged part in the first channel image (the leftmost one in Fig. 4(a)) with noise of $\sigma=30$ is shown in Fig. 5(a) and its output of PCA-UWT-CBF is shown in Fig. 5(b). An 


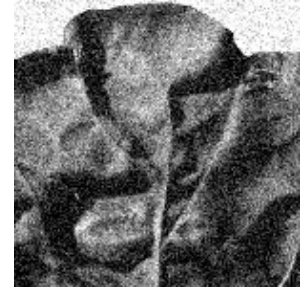

(a) noisy leaf

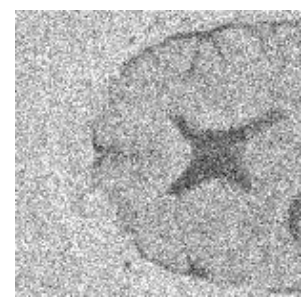

(c) noisy brain

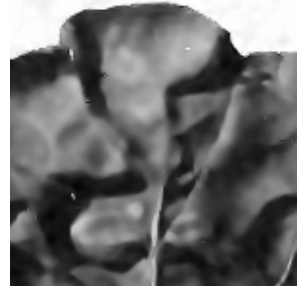

(b) PCA-IJWT-CBF

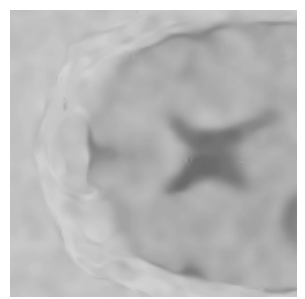

(d) PCA-UWT-CBF
Fig.5 Enlarged parts in input and output multi-modal images.

Table 4 Parameter values.

\begin{tabular}{|r|r|r|r|r|r|}
\hline & IBF & EBF & P-CBF & P-BF-CBF & P-WT-CBF \\
\hline$\sigma_{d}$ & 1.5 & 2 & 2 & $1.4,4.4$ & 4 \\
\hline$\sigma_{r}$ & 140 & 140 & 110 & 135,26 & 18 \\
\hline
\end{tabular}

\begin{tabular}{|r|r|r|r|r|r|}
\hline & IBF & EBF & P-CBF & P-BF-CBF & P-WT-CBF \\
\hline$\sigma_{d}$ & 4 & 3 & 3 & 2,2 & 2 \\
\hline$\sigma_{r}$ & 95 & 120 & 100 & 145,22 & 18 \\
\hline
\end{tabular}

(b) leaf

\begin{tabular}{|r|r|r|r|r|r|}
\hline & IBF & EBF & P-CBF & P-BF-CBF & P-WT-CBF \\
\hline$\sigma_{d}$ & 2 & 2 & 2 & $2,2.4$ & 2.6 \\
\hline$\sigma_{r}$ & 150 & 180 & 160 & 180,20 & 12 \\
\hline
\end{tabular}

(c) brain

enlarged part in the second channel image (the right one in Fig. 4(b)) with noise of $\sigma=30$ is shown in Fig. 5(c) and its output of PCA-UWT-CBF is shown in Fig. 5(d).

The values of parameters $\sigma_{d}$ and $\sigma_{r}$ are shown in Ta- ble 4 in the above experiments with $\sigma=30$ of which output images are shown in Fig. 2, Fig. 3 and Fig. 5. In the PBF-CBF in this table, two values are shown for $\sigma_{d}$ and $\sigma_{r}$, the first is that for $\mathrm{BF}$ and the second is for CBF. In the PWT-CBF, we used the UWT of 4 levels decomposition with global hard thresholding of $3.6 \times \sigma$. We always set $p=10$, i.e. window size is $21 \times 21$ in all experiments.

Note again that these values of parameters in each filter are optimal ones in the sense that they give the largest PSNR of each filter. Generally their optimal values are unknown. In such cases, the estimation of noise levels using MAD and adjustment of the value of parameters based on the estimated noise level may be effective practically. However in the experiments mentioned above, PSNR cannot exceed the values reported here even if we use any optimization method because the above PSNR are the largest ones.

\section{Conclusion}

We have presented a self-cross bilateral filter with a supplementary image generated using the PCA and demonstrated its effectiveness for denoising multi-modal images. Among some preliminary filters for the PCA-preF-CBF, the PCAUWT-CBF is shown to be useful for various types of images. Its further test for other practical multi-modal images with variable conditions is a subject of future research.

\section{References}

[1] C. Jian, K. Inoue, and K. Urahama, "Bootstrap denoising of images with self-cross bilateral filter," Proc. FCV, pp.62-65, 2010.

[2] C. Tomasi and R. Manduchi, "Bilateral filtering for gray and color images," Proc. ICCV, pp.839-846, 1998.

[3] E. Eisemann and D. Durand, "Flash photography enhancement via intrinsic relighting," ACM Trans. Graph., vol.23, no.3, pp.673-678, 2004.

[4] J.L. Starck, J. Fadili, and F. Murtagh, "The undecimated wavelet decomposition and its reconstruction," IEEE Trans. Image Process., vol.16, no.2, pp.297-309, 2007. 